\title{
APLICAÇÃO DO MÉTODO DA MÉDIA NO VOLUME NA MODELAGEM MATEMÁTICA DE UMA COLUNA ADSORÇÃO DE LEITO FIXO UTILIZANDO ISOTERMA COMPETITIVA
}

\author{
C. da LUZ ${ }^{1}$, A. D. da LUZ ${ }^{2}$, B. D. WOOD ${ }^{3}$, A. A. ULSON DE SOUZA ${ }^{4}$ e S. M. A. GUELLI U. \\ DE SOUZA ${ }^{4}$ \\ ${ }^{1}$ Universidade do Estado de Santa Catarina, Departamento de Engenharia de Alimentos \\ ${ }^{2}$ Universidade Federal da Fronteira Sul, Curso de Engenharia Ambiental \\ ${ }^{3}$ Oregon State University, School of Chemical, Biological, Environmental Engineering - USA \\ ${ }^{4}$ Universidade Federal de Santa Catarina, Departamento de Engenharia Química e de Alimentos \\ E-mails para contato: cleuzir@udesc.br, selene@enq.ufsc.br
}

\begin{abstract}
RESUMO - Geralmente as misturas submetidas ao processo de separação por adsorção usam adsorventes caracterizados por meios porosos com diferentes escalas de comprimento. Quando existe uma disparidade entre estas escalas, o Método da Média no Volume pode ser usado na modelagem matemática carregando, hierarquicamente, as informações físicas entre as escalas. Neste trabalho é usada a isoterma de Langmuir competitiva e é aplicado o Método da Média no Volume para obter um modelo de duas equações em concentrações médias volumétricas para fases sólida e líquida. O modelo possui informações físicas da microescala e macroescala do adsorvente; são encontradas expressões teóricas para os tensores de difusividade efetiva, dispersão total, convectivo de transferência de massa e tensores cruzados. Estes tensores de transporte podem ser obtidos mediante a resolução dos chamados problemas de fechamento, evitando assim a necessidade de utilização de parâmetros ajustáveis na simulação do processo. Neste trabalho é apresentada a resolução numérica de um problema de fechamento que permite calcular o tensor de difusividade efetiva, o qual carrega informações físicas da microescala.
\end{abstract}

\section{INTRODUÇÃ̃O}

Adsorção é um fenômeno de superfície no qual um soluto é removido de uma fase fluida e acumulado na superfície de uma fase sólida. O material adsorvido é denominado de adsorbato, e o material, sobre o qual o soluto é depositado, é chamado de adsorvente (Ruthven, 1984). O adsorvente geralmente é composto de micropartículas porosas que são empacotadas em um leito fixo por onde passa a fase fluida continuamente até que não haja mais transferência de massa. Uma vez que o adsorbato concentra-se na superfície do adsorvente, quanto maior for esta superfície, maior será a eficiência da adsorção. Para abordar a adsorção de um soluto com multicomponentes este trabalho apresenta uma modelagem matemática com isoterma de Langmuir competitiva que descreve o processo de adsorção em uma coluna em leito fixo, aplicando o Método da Média no Volume descrito por Whitaker (1985). Este método permite fazer a Modelagem Matemática em diferentes escalas do adsorvente, carregando informações fenomenológicas desde a microescala até a macroescala, conforme mostrado na Figura 1. 


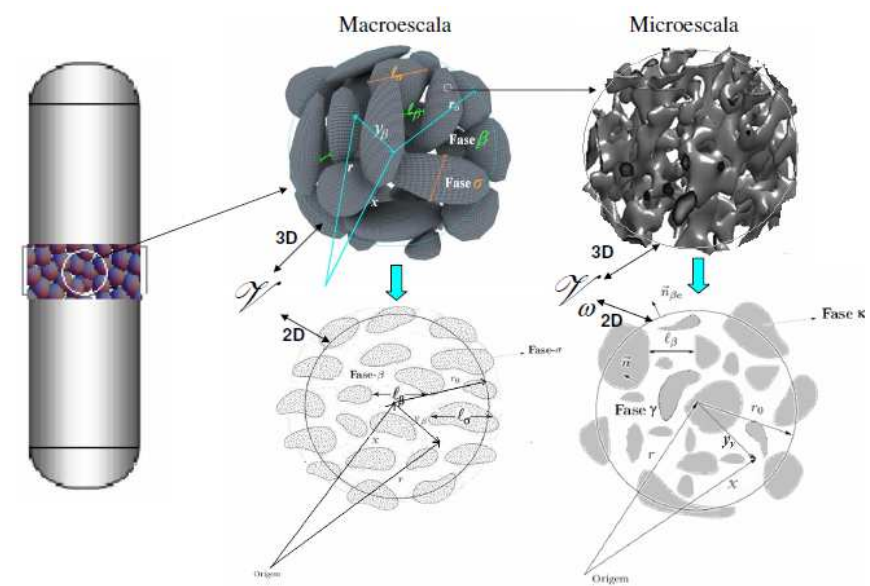

Figura 1 - Hierarquia de escalas de comprimento no meio poroso de uma coluna de adsorção.

\section{MODELAGEM MATEMÁTICA}

\subsection{Modelagem Matemática da Microescala}

A modelagem matemática da microescala do adsorvente poroso é feita a partir de um volume de controle $\mathscr{Q}_{\omega}$ da microescala. A microescala é formada pela fase sólida do adsorvente impermeável, denominada de fase $k$, e pela fase fluida contida nos poros do adsorvente, denominada de fase $\gamma$, conforme ilustrado na Figura 1. O vetor $\boldsymbol{r}$ localiza qualquer ponto no espaço tridimensional $\left(\mathbb{R}^{3}\right)$, o vetor $\boldsymbol{r}_{\boldsymbol{o}}$ é o raio de $\mathscr{V}_{\omega}$, o vetor posição $\boldsymbol{x}$ determina o centroide do $\mathscr{T}_{\omega}$, o vetor posição $\boldsymbol{y}_{\gamma}$ localiza os pontos na fase $\gamma$ em relação ao centroide do $\mathscr{C}_{\omega}, \ell_{\gamma} \mathrm{e} \ell_{\kappa}$ representam os comprimentos característicos das fases. A interface $\mathscr{C} / \gamma_{k}$ é a superfície das paredes impermeáveis do adsorvente, onde a adsorção das espécies pode ocorrer e a interface Che são as superfícies das entradas e saídas da fase contínua. As equações de transporte governantes e condições de contorno interfaciais que representam o processo de adsorção no volume de controle $\mathscr{Q}_{\omega}$ são dadas por:

$$
\begin{aligned}
& \frac{\partial C_{i \gamma}}{\partial t}=\nabla \cdot\left(\mathscr{O}_{i \gamma} \nabla C_{i \gamma}\right), \quad \text { na fase } \gamma \\
& \text { C.C.1 }-\boldsymbol{n}_{\gamma \kappa} \cdot \sigma_{i \gamma} \nabla C_{i \gamma}=\frac{\partial C_{S i}}{\partial t}, \quad \text { em } \quad \mathscr{d}_{\gamma \kappa} \quad \text { e } \quad C_{S i}=\frac{K_{i} b_{i} C_{i}}{1+b_{1} C_{1}+b_{2} C_{2}+b_{3} C_{3}} \\
& \text { C.C.2 } C_{i \gamma}=\mathscr{T}_{i \gamma}(t, r), \quad \text { em } d C_{\gamma e} \\
& \begin{array}{ll}
\text { C.I. } C_{i \gamma}=\mathscr{C}_{i \gamma}(r), \quad \mathrm{em} \mathrm{t}=0,
\end{array}
\end{aligned}
$$

em que $C_{i \gamma}$ é a concentração pontual na fase $\gamma$ do $\mathscr{T}_{\omega}$ das espécies $i$, $\mathscr{T}_{i \gamma}$ é a difusividade molecular da espécie $i, \boldsymbol{n}_{\gamma \kappa}$ é o vetor unitário normal à área $\mathscr{A}_{\gamma \kappa}$ e $C_{S i}$ é a concentração de superfície da espécie $i$. Sendo um processo de adsorção, a C.C.1 dada pela Equação (2.02), é 


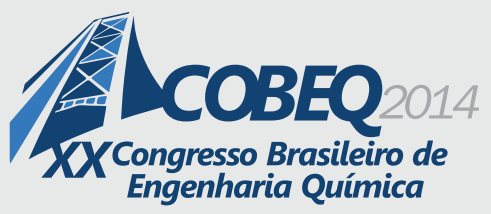

baseada na isoterma de Langmuir competitiva, tal que $K_{i}$ é o coeficiente de equilíbrio de adsorção e $b_{i}$ são as constantes de Langmuir das espécies químicas $i: 1,2,3$. A C.C.2 dada pela Equação (2.03) mostra que a concentração pontual em $\mathscr{C}_{\gamma e}$ é dada pela função $\mathscr{\mathscr { C } _ { i \gamma }}(t, r)$ e a $C . I$ na Equação (2.04) é dada pela função $\mathscr{C}(r)$.

Método da Média no Volume: O problema apresentado nas Equações (2.01) à (2.04) não pode ser resolvido da forma que se encontra, pois não se conhecem as funções $\mathscr{\mathscr { F } _ { i \gamma }}(t, r)$ e $\mathscr{C}(r)$; mesmo as conhecendo, tal solução apresentaria mais informações do que são realmente necessárias. O objetivo é utilizar as informações da microescala para compor equações governantes na macroescala. Para isso é aplicado o Método da Média no Volume, que consiste em transformar a concentração pontual, em uma concentração média para todo $\mathscr{V}_{\omega}$, que é a soma

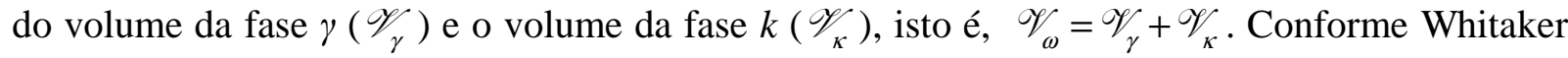
(1999) este método utiliza a Concentração Média Volumétrica Superficial e a Concentração Média Intrínseca, Equações (2.05), respectivamente. Uma relação importante entre estas é dada por $\left\langle C_{i \gamma}\right\rangle=\varepsilon_{\gamma}\left\langle C_{i \gamma}\right\rangle^{\gamma}$, em que $\varepsilon_{\gamma}$ é a porosidade da fase $\gamma\left(\varepsilon_{\gamma}=q_{\gamma} / q_{\omega}\right)$.

$$
\left\langle C_{i \gamma}\right\rangle=\frac{1}{q \omega} \int_{V_{\gamma}} C_{i \gamma} d V \text { e }\left\langle C_{i \gamma}\right\rangle^{\gamma}=\frac{1}{q \gamma} \int_{V_{\gamma}} C_{i \gamma} d V
$$

Análogo aos procedimentos matemáticos de Whitaker (1999) - Cap. 1, faz-se o processo de suavização espacial com a importante aplicação do Teorema da Média Espacial, mostrado no estudo de Howes e Whitaker (1985). A Aplicação deste Teorema ilustra um aspecto fundamental do processo de suavização espacial, onde a C.C.l é introduzida na equação governante através da integral do fluxo. Assim chega-se a Equação (2.06):

$$
\varepsilon_{\gamma} \frac{\partial\left\langle C_{i \gamma}\right\rangle^{\gamma}}{\partial t}=\nabla \cdot[\overbrace{i \gamma}\left(\varepsilon_{\gamma} \nabla\left\langle C_{i \gamma}\right\rangle^{\gamma}+\frac{1}{y_{\omega}} \int_{A_{A_{\mu}}} \boldsymbol{n}_{\gamma \kappa} \tilde{C}_{i \gamma} d A\right)]-\left.a_{\nu}\right|_{\gamma \kappa} \frac{\partial}{\partial t}\left(\frac{\left.K_{i} b_{i}\left\langle C_{i}\right\rangle^{\gamma}\right|_{x}}{1+\left.b_{1}\left\langle C_{1}\right\rangle^{\gamma}\right|_{x}+\left.b_{2}\left\langle C_{2}\right\rangle^{\gamma}\right|_{x}+\left.b_{3}\left\langle C_{3}\right\rangle^{\gamma}\right|_{x}}\right) .
$$

Para obtenção da Equação (2.06) considerou-se o estudo de Gray (1975) que sugere uma decomposição espacial da concentração pontual, primeira identidade de (2.07), e também foi provada a validade da segunda identidade de (2.07).

$$
C_{i \gamma}=\left\langle C_{i \gamma}\right\rangle^{\gamma}+\tilde{C}_{i \gamma} \text { e }\left\langle C_{S i}\right\rangle_{\gamma \kappa}=\left.\left\langle C_{S i}\right\rangle^{\gamma}\right|_{x}
$$

Considerou-se válidas as identidades da teoria geométrica e a definição de meio desordenado de Quintard e Whitaker (1994). Desde que válidas as restrições de comprimento de escala

$$
\left(\frac{r_{o}}{L_{c}}\right) \ll 1,\left(\frac{r_{o}^{2}}{L_{\varepsilon} L_{c 1}}\right) \ll<1, \ell_{\gamma} \ll r_{o} \text { e }\left\langle\boldsymbol{y}_{\gamma}\right\rangle_{\gamma \kappa} \ll r_{o} \text {. }
$$

Problema de Fechamento na Microescala: O chamado problema de fechamento é a forma de encontrar uma representação matemática de $\tilde{C}_{i \gamma}$ em termos de $\left\langle C_{i \gamma}\right\rangle^{\gamma}$ na Equação (2.06), obtendo a forma fechada da equação governante da microescala. Este problema é obtido subtraindo-se, Equação (2.06) da Equação (2.01) e assumindo as restrições de ordem de grandeza 


$$
\frac{\ell_{\gamma}}{L_{c}}<1 \text { e } \frac{\mathscr{P}_{\gamma} t^{*}}{\ell_{\gamma}^{2}}>>1 .
$$

Conforme estudos de Ryan et al., (1981) e Quintard e Whitaker, (1993) pode-se propor a solução do problema de fechamento em uma região representativa e substituir as condição de contorno que não são conhecidas por condições periódicas, chegando ao problema de fechamento:

$$
\begin{aligned}
& \nabla^{2} \tilde{C}_{i \gamma}=-\left(\frac{\left.a_{v}\right|_{\gamma \kappa} K_{i}}{\varepsilon_{\gamma} \Im_{i \gamma}}\right) \frac{\partial}{\partial t}\left(\frac{\left.b_{i}\left\langle C_{i}\right\rangle^{\gamma}\right|_{x}}{1+\left.b_{1}\left\langle C_{1}\right\rangle^{\gamma}\right|_{x}+\left.b_{2}\left\langle C_{2}\right\rangle^{\gamma}\right|_{x}+\left.b_{3}\left\langle C_{3}\right\rangle^{\gamma}\right|_{x}}\right)
\end{aligned}
$$

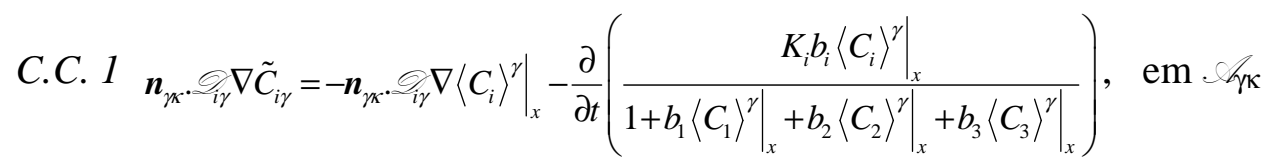

$$
\begin{aligned}
& \text { C.C.2 } \tilde{C}_{i \gamma}\left(r+\ell_{j}\right)=\tilde{C}_{i \gamma}(r), \quad j=1,2,3 .
\end{aligned}
$$

Pelo Princípio da Superposição, propõe-se, uma solução do Problema de Fechamento Local dado pelas Equações (2.10) a (2.12), da forma:

$$
\tilde{C}_{i \gamma}=\left.\boldsymbol{b}_{i \gamma} \cdot \nabla\left\langle C_{i \gamma}\right\rangle^{\gamma}\right|_{x}+s_{i \gamma} \frac{\partial}{\partial t}\left(\frac{\left.b_{i}\left\langle C_{i}\right\rangle^{\gamma}\right|_{x}}{1+\left.b_{1}\left\langle C_{1}\right\rangle^{\gamma}\right|_{x}+\left.b_{2}\left\langle C_{2}\right\rangle^{\gamma}\right|_{x}+\left.b_{3}\left\langle C_{3}\right\rangle^{\gamma}\right|_{x}}\right)+\psi_{i \gamma}
$$

Em que $\boldsymbol{b}_{i \gamma}, s_{i \gamma}$ e $\psi_{i \gamma}$ são as variáveis de fechamento e soluções dos chamados problemas de fechamento, porém neste caso os problemas de valor de contorno relacionados a $s_{i \gamma}$ e $\psi_{i \gamma}$ são negligenciados, conforme Whitaker (1999) - Cap. 1. Portanto apresenta-se somente o problema: Problema I $: \nabla^{2} \boldsymbol{b}_{i \gamma}=0$

$$
\text { C.C.1 }-\boldsymbol{n}_{\gamma \kappa} \cdot \nabla \boldsymbol{b}_{i \gamma}=\boldsymbol{n}_{\gamma \kappa} \text { em . } / \gamma_{\kappa} \text { e Periodicidade: } \boldsymbol{b}_{i \gamma}\left(\boldsymbol{r}+\ell_{j}\right)=\boldsymbol{b}_{i \gamma}(\boldsymbol{r}), j=1,2,3
$$

Forma Fechada da Equação para Microescala: Substituindo a solução do problema de fechamento, Equação (2.13) na Equação (2.06), chega-se à forma fechada,

$$
\varepsilon_{\gamma} \frac{\partial\left\langle C_{i \gamma}\right\rangle^{\gamma}}{\partial t}=\nabla \cdot\left(\left.\varepsilon_{\gamma} \boldsymbol{D}_{e f f i}\right|_{\gamma \kappa} \cdot \nabla\left\langle C_{i \gamma}\right\rangle^{\gamma}\right)-\left.a_{v}\right|_{\gamma \kappa} K_{i} \frac{\partial}{\partial t}\left(\frac{\left.b_{i}\left\langle C_{i}\right\rangle^{\gamma}\right|_{x}}{1+\left.b_{1}\left\langle C_{1}\right\rangle^{\gamma}\right|_{x}+\left.b_{2}\left\langle C_{2}\right\rangle^{\gamma}\right|_{x}+\left.b_{3}\left\langle C_{3}\right\rangle^{\gamma}\right|_{x}}\right),
$$

em que $\left.\boldsymbol{D}_{\text {eff } i}\right|_{\gamma \kappa}=\mathscr{Q}_{i \gamma}\left(\boldsymbol{I}+\frac{1}{q / \gamma} \int_{\partial \gamma_{\gamma \kappa}} \boldsymbol{n}_{\gamma k} \boldsymbol{b}_{i \gamma} d A\right)$ é o tensor difusividade efetiva da espécie $i$.

\subsection{Modelagem Matemática da Macroescala}

Na Figura 1, y é formado por macroporos contendo uma fase fluida (fase $\beta$ ), e de uma região microporosa (região $\sigma$ ). O raio do $y^{\prime}$ é representado por $r_{0}$ e os símbolos $\ell_{\beta} \mathrm{e} \ell_{\sigma}$ são usados para representar os comprimentos característicos das fases. O problema de difusão e adsorção é dado pelas equações diferenciais e condições de contorno interfaciais: 


$$
\begin{aligned}
& \frac{\partial C_{i \beta}}{\partial t}+\nabla \cdot\left(v_{\beta} C_{i \beta}\right)=\nabla \cdot\left(\mathscr{C}_{i \beta} \nabla C_{i \beta}\right) \quad \text {, na fase } \beta
\end{aligned}
$$

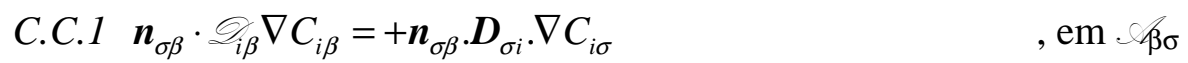

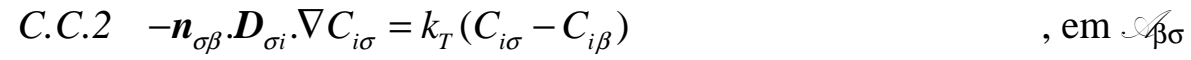

$$
\begin{aligned}
& \varepsilon_{\gamma} \frac{\partial C_{i \sigma}}{\partial t}=\nabla \cdot\left(\left.\varepsilon_{\gamma} \boldsymbol{D}_{e f f i}\right|_{\gamma \kappa} . \nabla C_{i \sigma}\right)-\left.a_{v}\right|_{\gamma \kappa} \frac{\partial}{\partial t}\left(\frac{K_{i} b_{i} C_{i \sigma}}{1+b_{1} C_{1 \sigma}+b_{2} C_{2 \sigma}+b_{3} C_{3 \sigma}}\right) \text {, na região } \sigma \\
& \text { C.C.3 } C_{i \beta}=\mathscr{O}_{i}(r, t) \quad \mathrm{em} / \mathrm{\beta e}_{\mathrm{e}} \text { e C.C.4 } C_{i \sigma}=\mathscr{C}_{i}(r, t) \text {, em } / \sigma \mathrm{e} \\
& \text { C.I.I } C_{i \beta}=\mathscr{L}_{i}(r) \quad \mathrm{em} \mathrm{t}=0 \text { e C.I.2 } C_{i \sigma}=\mathscr{C}_{i}(r) \quad, \mathrm{em} \mathrm{t}=0 .
\end{aligned}
$$

Em que $\boldsymbol{D}_{i \sigma}=\left.\varepsilon_{\gamma} \boldsymbol{D}_{e f i}\right|_{\gamma \kappa}, k_{T}$ é o coeficiente de transferência de massa e $\left\langle C_{i \gamma}\right\rangle^{\gamma}=C_{i \sigma}$. Analogamente à modelagem matemática da microescala, aplica-se o Método da Média no Volume nas equações pontuais do campo de concentração para fase $\beta$ e para região $\sigma$, chegandose a um Problema de Fechamento, o qual pelo Princípio da Superposição, tem-se as soluções:

$$
\begin{aligned}
& \tilde{C}_{i \beta}=\boldsymbol{b}_{i \beta \beta} \cdot \nabla\left\langle C_{i \beta}\right\rangle^{\beta}+\boldsymbol{b}_{i \beta \sigma} \cdot \nabla\left\langle C_{i \sigma}\right\rangle^{\sigma}+t_{i \beta}\left(\left\langle C_{i \sigma}\right\rangle^{\sigma}-\left\langle C_{i \beta}\right\rangle^{\beta}\right)+\varphi_{i \beta} \\
& \tilde{C}_{i \sigma}=\boldsymbol{b}_{i \sigma \beta} \cdot \nabla\left\langle C_{i \beta}\right\rangle^{\beta}+\boldsymbol{b}_{i \sigma \sigma} \cdot \nabla\left\langle C_{i \sigma}\right\rangle^{\sigma}+t_{i \sigma}\left(\left\langle C_{i \sigma}\right\rangle^{\sigma}-\left\langle C_{i \beta}\right\rangle^{\beta}\right)+\varphi_{i \sigma}
\end{aligned}
$$

em que $\boldsymbol{b}_{i \beta \beta}, \boldsymbol{b}_{i \beta \sigma}, \boldsymbol{b}_{i \sigma \sigma}, \boldsymbol{b}_{i \sigma \beta}, t_{i \beta}, t_{i \sigma}, \varphi_{i \beta}$ e $\varphi_{i \sigma}$ são denominadas variáveis de fechamento e são soluções dos respectivos problemas de valor de contorno:

Problema I: $\boldsymbol{v}_{\beta} \cdot \nabla \boldsymbol{b}_{i \beta \beta}+\tilde{\boldsymbol{v}}_{\beta}=\nabla \cdot(\overbrace{i \beta} \nabla \boldsymbol{b}_{i \beta \beta})-\frac{\varepsilon_{\beta}^{-1}}{q \sigma} \int_{\beta \sigma \sigma} \boldsymbol{n}_{\beta \sigma} \cdot \overbrace{i \beta} \nabla \boldsymbol{b}_{i \beta \beta} d A$, na região $\beta$

$$
\text { C.C.1 }-\boldsymbol{n}_{\sigma \beta} . \boldsymbol{D}_{i \sigma} \nabla \boldsymbol{b}_{\sigma \beta}=0 \text { e C.C.2 } \boldsymbol{n}_{\beta \sigma} \cdot \mathscr{O}_{i \beta}+\boldsymbol{n}_{\beta \sigma} \cdot{ }_{i \beta} \nabla \boldsymbol{b}_{i \beta \beta}=\boldsymbol{n}_{\beta \sigma} \cdot \boldsymbol{D}_{i \sigma} \cdot \boldsymbol{b}_{i \sigma \beta}
$$

$$
\nabla \cdot\left(\boldsymbol{D}_{i \sigma} \cdot \nabla \boldsymbol{b}_{i \sigma \beta}\right)=\frac{\varepsilon_{\sigma}^{-1}}{q} \cdot \int_{A_{\beta \sigma}} \boldsymbol{n}_{\sigma \beta} \cdot \boldsymbol{D}_{i \sigma} \cdot \nabla \boldsymbol{b}_{i \sigma \beta} d A, \quad \text { na região } \sigma
$$

Periodicidade: $\boldsymbol{b}_{i \beta \beta}\left(\boldsymbol{r}+\ell_{j}\right)=\boldsymbol{b}_{i \beta \beta}(\boldsymbol{r})$ e $\boldsymbol{b}_{i \sigma \beta}\left(\boldsymbol{r}+\ell_{j}\right)=\boldsymbol{b}_{i \sigma \beta}(\boldsymbol{r}), \quad j=1,2,3$

Problema II: $\boldsymbol{v}_{\beta} \cdot \nabla \boldsymbol{b}_{i \beta \sigma}=\nabla \cdot\left(\mathscr{T}_{i \beta} \nabla \boldsymbol{b}_{i \beta \sigma}\right)-\frac{\boldsymbol{\varepsilon}_{\beta}^{-1}}{\vartheta^{\prime}} \int_{A_{\beta \sigma}} \boldsymbol{n}_{\beta \sigma} \cdot \mathscr{C}_{i \beta} \nabla \boldsymbol{b}_{i \beta \sigma} d A$, na região $\beta$

$$
\text { C.C.1 }-\boldsymbol{n}_{\sigma \beta} . \boldsymbol{D}_{i \sigma}-\boldsymbol{n}_{\sigma \beta} . \boldsymbol{D}_{i \sigma} \nabla \boldsymbol{b}_{\sigma \sigma}=0
$$

C.C. $2 \boldsymbol{n}_{\beta \sigma} \cdot \mathscr{T}_{i \beta} \nabla \boldsymbol{b}_{i \beta \sigma}=\boldsymbol{n}_{\beta \sigma} . D_{i \sigma}+\boldsymbol{n}_{\beta \sigma} . D_{i \sigma} . \nabla b_{i \sigma \sigma}$

$$
\nabla \cdot\left(\boldsymbol{D}_{i \sigma} . \nabla \boldsymbol{b}_{i \sigma \sigma}\right)=\frac{\varepsilon_{\sigma}^{-1}}{q} \int_{A_{\beta \sigma}} \boldsymbol{n}_{\sigma \beta} \cdot \boldsymbol{D}_{i \sigma} . \nabla \boldsymbol{b}_{i \sigma \sigma} d A, \quad \text { na região } \sigma
$$

Periodicidade: $\boldsymbol{b}_{i \beta \sigma}\left(\boldsymbol{r}+\ell_{j}\right)=\boldsymbol{b}_{i \beta \sigma}(\boldsymbol{r})$ e $\boldsymbol{b}_{i \sigma \sigma}\left(\boldsymbol{r}+\ell_{j}\right)=\boldsymbol{b}_{i \sigma \sigma}(\boldsymbol{r}), \quad j=1,2,3$ 
Problema III: $\boldsymbol{v}_{\beta} \cdot \nabla t_{i \beta}=\nabla \cdot\left(\mathscr{C}_{i \beta} \nabla t_{i \beta}\right)-\frac{\varepsilon_{\beta}^{-1}}{y} \int_{\sigma_{\beta \sigma}} \boldsymbol{n}_{\beta \sigma} \cdot \overbrace{i \beta} \nabla t_{i \beta} d A, \quad$ na região $\beta$

C.C. $1-\boldsymbol{n}_{\sigma \beta} . \boldsymbol{D}_{i \sigma} \cdot \nabla t_{i \beta}=k_{T} \quad$ e $\quad$ C.C. $2 \boldsymbol{n}_{\beta \sigma} \cdot \mathscr{O}_{i \beta} \nabla t_{i \beta}=\boldsymbol{n}_{\beta \sigma} \cdot \boldsymbol{D}_{i \sigma} \cdot \nabla t_{i \beta}$

$\nabla \cdot\left(\boldsymbol{D}_{i \sigma} \cdot \nabla t_{i \beta}\right)=\frac{\boldsymbol{\varepsilon}_{\sigma}^{-1}}{q} \int_{A_{\sigma \beta}} \boldsymbol{n}_{\sigma \beta} \cdot \boldsymbol{D}_{i \sigma} \cdot \nabla t_{i \beta} d A$,

na região $\sigma$

Periodicidade: $t_{i \beta}\left(\boldsymbol{r}+\ell_{j}\right)=t_{i \beta}(\boldsymbol{r})$ e $t_{i \sigma}\left(\boldsymbol{r}+\ell_{j}\right)=t_{i \sigma}(\boldsymbol{r})$

$j=1,2,3$

Forma Fechada: Com as soluções dos problemas de fechamento dadas pelas Equações (2.24) e (2.25), chega-se à forma fechada do modelo de duas equações:

$$
\begin{aligned}
& \mathcal{E}_{\beta} \frac{\partial\left\langle C_{i \beta}\right\rangle^{\beta}}{\partial t}=\nabla \cdot\left(\boldsymbol{D}_{i \beta \beta}^{*} \cdot \nabla\left\langle C_{i \beta}\right\rangle^{\beta}\right)+\nabla \cdot\left(\boldsymbol{D}_{i \beta \sigma}^{*} \cdot \nabla\left\langle C_{i \sigma}\right\rangle^{\sigma}\right) \\
& +\nabla \cdot\left[\boldsymbol{u}_{i \beta} k_{T}\left(\left\langle C_{i \sigma}\right\rangle^{\sigma}-\left\langle C_{i \beta}\right\rangle^{\beta}\right)\right]+\left.a_{v}\right|_{\beta \sigma} k_{T}\left(\left\langle C_{i \sigma}\right\rangle^{\sigma}-\left\langle C_{i \beta}\right\rangle^{\beta}\right)-\nabla \cdot\left(\varepsilon_{\beta}\left\langle\boldsymbol{v}_{\beta i}\right\rangle^{\beta}\left\langle C_{i \beta}\right\rangle^{\beta}\right) \\
& \boldsymbol{D}_{i \beta \beta}^{*}=\mathscr{C}_{i \beta} \varepsilon_{\beta}\left(\boldsymbol{I}+\frac{1}{q_{\beta}} \int_{\beta / \beta \sigma} \boldsymbol{n}_{\beta \sigma} \cdot \boldsymbol{b}_{i \beta \beta} d A\right)-\left\langle\tilde{\boldsymbol{v}}_{\beta} \boldsymbol{b}_{i \beta \beta}\right\rangle, \boldsymbol{D}_{i \beta \sigma}^{*}=\mathscr{I}_{i \beta} \mathcal{E}_{\beta}\left(\frac{1}{\sigma_{\beta}} \int_{\beta / \beta \sigma} \boldsymbol{n}_{\beta \sigma} \boldsymbol{b}_{i \beta \beta} d A\right)-\left\langle\tilde{\boldsymbol{v}}_{\beta} \boldsymbol{b}_{i \beta \beta}\right\rangle \\
& \text { e } \boldsymbol{u}_{i \beta}=\frac{\mathscr{T}_{i \beta} \varepsilon_{\beta}}{k_{T}}\left(\frac{1}{q_{\beta}} \int_{\beta \sigma} \boldsymbol{n}_{\beta \sigma} t_{i \beta} d A\right)-\frac{1}{k_{T}}\left\langle\tilde{\boldsymbol{v}}_{\beta} t_{i \beta}\right\rangle \text {; } \\
& \varepsilon_{\sigma} \varepsilon_{\gamma} \frac{\partial\left\langle C_{i \sigma}\right\rangle^{\sigma}}{\partial t}=\nabla \cdot\left(\boldsymbol{D}_{i \sigma \sigma}^{*} . \nabla\left\langle C_{i \sigma}\right\rangle^{\sigma}\right)+\nabla \cdot\left(\boldsymbol{D}_{i \sigma \beta}^{*} \cdot \nabla\left\langle C_{i \beta}\right\rangle^{\beta}\right)+\nabla \cdot\left[\boldsymbol{u}_{i \sigma} k_{T}\left(\left\langle C_{i \sigma}\right\rangle^{\sigma}-\left\langle C_{i \beta}\right\rangle^{\beta}\right)\right] \\
& -\left.a_{v}\right|_{\beta \sigma} k_{T}\left(\left\langle C_{i \sigma}\right\rangle^{\sigma}-\left\langle C_{i \beta}\right\rangle^{\beta}\right)-\left.\varepsilon_{\sigma} a_{v}\right|_{\gamma \kappa} \frac{\partial}{\partial t}\left(\frac{K_{i} b_{i}\left\langle C_{i \sigma}\right\rangle^{\sigma}}{1+b_{1}\left\langle C_{1 \sigma}\right\rangle^{\sigma}+b_{2}\left\langle C_{2 \sigma}\right\rangle^{\sigma}+b_{3}\left\langle C_{3 \sigma}\right\rangle^{\sigma}}\right) \\
& \boldsymbol{D}_{i \sigma \beta}^{*}=\varepsilon_{\sigma}\left(\frac{\boldsymbol{D}_{i \sigma}}{\sigma \sigma} \cdot \int_{\gamma \beta} \boldsymbol{n}_{\sigma \beta} \boldsymbol{b}_{i \sigma \beta} d A\right), \boldsymbol{D}_{i \sigma \sigma}^{*}=\varepsilon_{\sigma}\left(\boldsymbol{D}_{i \sigma}+\frac{\boldsymbol{D}_{i \sigma}}{\sigma_{\beta}} \cdot \int_{\beta \sigma \sigma} \boldsymbol{n}_{\sigma \beta} \boldsymbol{b}_{i \sigma \sigma} d A\right) \text { e } \boldsymbol{u}_{i \sigma}=\frac{\varepsilon_{\sigma}}{k_{T}}\left(\frac{\boldsymbol{D}_{i \sigma}}{\sigma / \sigma} \cdot \int_{i \beta \sigma} \boldsymbol{n}_{\sigma \beta} t_{i \sigma} d A\right)
\end{aligned}
$$

Estes tensores de transporte são tais como: tensor de difusividade efetiva da região $\sigma, \boldsymbol{D}_{i \sigma \sigma}^{*}$, tensor de dispersão total da fase $\beta, \boldsymbol{D}_{i \beta \beta}^{*}$, tensores cruzados $\boldsymbol{D}_{i \beta \sigma}^{*}$ e $\boldsymbol{D}_{i \sigma \beta}^{*}$ e coeficientes $\boldsymbol{u}_{i \sigma}$ e $\boldsymbol{u}_{i \beta}$.

\section{RESULTADOS E DISCUSSÃO}

Neste trabalho são apresentados os resultados numéricos do $\left.\boldsymbol{D}_{\text {eff } i}\right|_{{ }_{\digamma}}$, tensor de difusividade efetiva, que carrega as informações físicas da microescala para macroescala através do $\boldsymbol{D}_{i \sigma}$. O Método de Volumes Finitos foi utilizado para discretizar o conjunto de equações do modelo e a resolução numérica foi realizada através do Software livre OpenFoam V2.1.1. Na Figura 2 (a)-(e) são apresentados os perfis de $\boldsymbol{b}_{i \gamma}$ para $\varepsilon_{\gamma}=0,50$ sobre arranjos de cilindros inteiros e para $\varepsilon_{\gamma}=0,73$ sobre $1 / 8$ de esferas. 
(a)

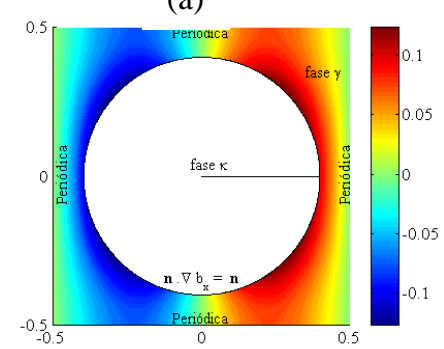

(b)

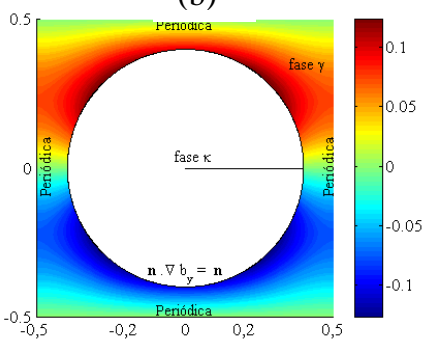

(d)
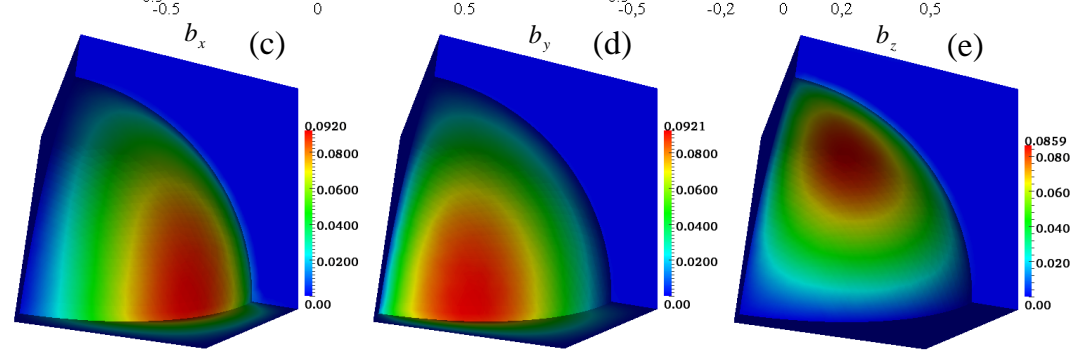

Figura 2 - Perfis de $b_{x}, b_{y}$ e $b_{z}$ em arranjos de cilindros inteiros e 1/8 esferas.

Pelas propriedades de simetria e isotropia é suficiente usar arranjos de 1/4 cilindros ou 1/8 de esferas para calcular a função escalar $b_{x}$. Na Figura 3 (a)-(c) são apresentadas as soluções numéricas obtidas neste trabalho, a solução analítica de Chang (1982), dados numéricos de Ochoa-Tapia e Whitaker (1994) e dados do modelo de Maxwell e Quintard e Whitaker (1993).

(a)

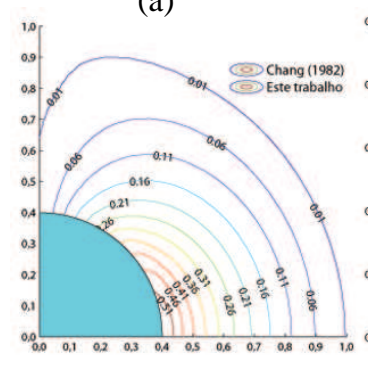

(b)

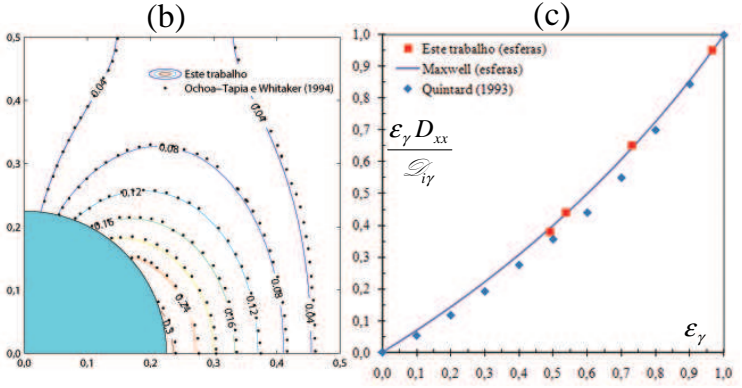

(d)

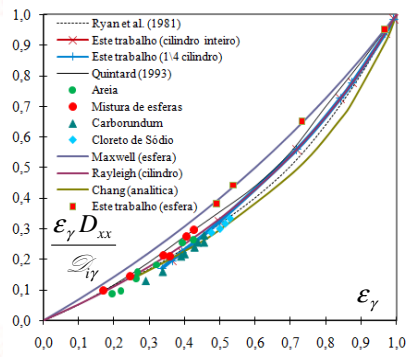

Figura 3 - Solução numérica deste trabalho comparada com: (a) Solução analítica de Chang (1982) calculada na

forma $2 b_{x} / \ell(\varepsilon=0,84$ ); (b) Solução numérica de Ochoa-Tapia e Whitaker (1994) calculada na forma

$2 b_{x} / \ell(\varepsilon=0,84$ ); (c) Quintard e Whitaker (1993) e Maxwell com outros valores de porosidade);

(d) Teóricos experimentais.

A Figura 3 (d) mostra que os resultados obtidos neste trabalho para o coeficiente de difusividade efetiva em várias porosidades, estão de acordo com resultados obtidos por outros autores e com os dados experimentais para arranjos de cilindros e esferas.

\section{CONCLUSÃO}

A realização da modelagem matemática aplicando o Método da Média no Volume forneceu o modelo de duas equações que descreve o transporte dos multicomponentes na escala de Darcy (escala de projeto). Esta modelagem, considerando a isoterma de Langmuir competitiva, 


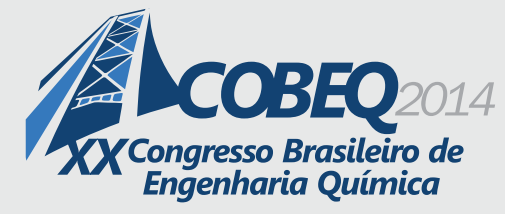

incorporou todas as informações fenomenológicas relevantes do processo de adsorção. Isso é um importante diferencial com relação aos modelos clássicos. O modelo é capaz de calcular as diversas resistências à transferência de massa durante o processo de adsorção. Os problemas de fechamento apresentados neste trabalho geram um conjunto de equações teóricas, que possibilitam posteriormente calcular analiticamente ou numericamente os tensores de transporte nas diferentes escalas. Foram apresentados resultados numéricos para o tensor de difusividade efetiva, o qual carrega informações da microescala para macroescala, verificando-se boa concordância entre resolução numérica deste trabalho com dados teóricos e experimentais.

\section{AGRADECIMENTOS}

Os autores agradecem ao apoio financeiro concedido pelo Conselho Nacional de Desenvolvimento Científico e Tecnológico - CNPq, ao LABSIN - Laboratório de Simulação Numérica e ao LABMASSA - Laboratório de Transferência de Massa, da UFSC.

\section{REFERÊNCIAS}

CHANG, H-C. Multi-scale analysis of effective transport in periodic heterogeneous media. Chemical Engineering Communications, v. 15, p. 83-91. 1982.

GRAY, W.G. A derivation of equations for multiphase transport. Chemical Engineering Science, v. 30, p.229-233, 1975.

HOWES, F. A.; WHITAKER, S. The spatial averaging theorem revisited. Chemical Engineering Science, v. 40, p.1387-1392, 1985.

OCHOA-TAPIA, J. A.; WHITAKER, S. Diffusive transport in two-phase media: spatially periodic models and Maxwell's theory for isotropic and anisotropic systems. Chemical Engineering Science, v. 49, n. 5, p. 709-726, 1994.

QUINTARD, M.; WHITAKER, S. Transport in ordered and disordered porous media: Volume averaged equations, closure problems, and comparison with experiment. Chemical Engineering Science, v. 48, p. 2537-2564, 1993.

QUINTARD, M.; WHITAKER, S. Transport in ordered and disordered porous media II: generalized volume averaging. Transport in Porous Media, v. 14, p. 179-206, 1994.

RUTHVEN, D. M. Principles of Adsorption and Adsorption Process, John Wiley \& Sons, New York, 432 p., 1984.

RYAN, D.; CARBONELL, R. G.; WHITAKER, S. A Theory of Diffusion and Reaction in Porous Media, AIChE Symposium Series, \#202, v. 71, p. 46-62, 1981.

WHITAKER, S. A Simple Geometrical Derivation of the Spatial Averaging Theorem, Chemical Engineering Education, v. 19, p. 18-21, 50-52, 1985.

WHITAKER, S. Theory and applications of transport in porous media: the method of volume averaging. London: Kluwer Academic, 1999. 